POS PROCEEDINGS

\title{
R-D perspectives for new neutrino beams at accelerators
}

\section{MAURIZIO BONESINI ${ }^{* \dagger}$}

Sezione INFN Milano Bicocca

E-mail: maurizio.bonesini@mib.infn.it

New developments for neutrino physics at accelerators are reviewed, with a special emphasis on superbeams, neutrino factories and their related R-D projects. Their physics reach will be outlined.

European Physical Society Europhysics Conference on High Energy Physics

July 16-22, 2009

Krakow, Poland

${ }^{*}$ Speaker.

${ }^{\dagger}$ A footnote may follow. 


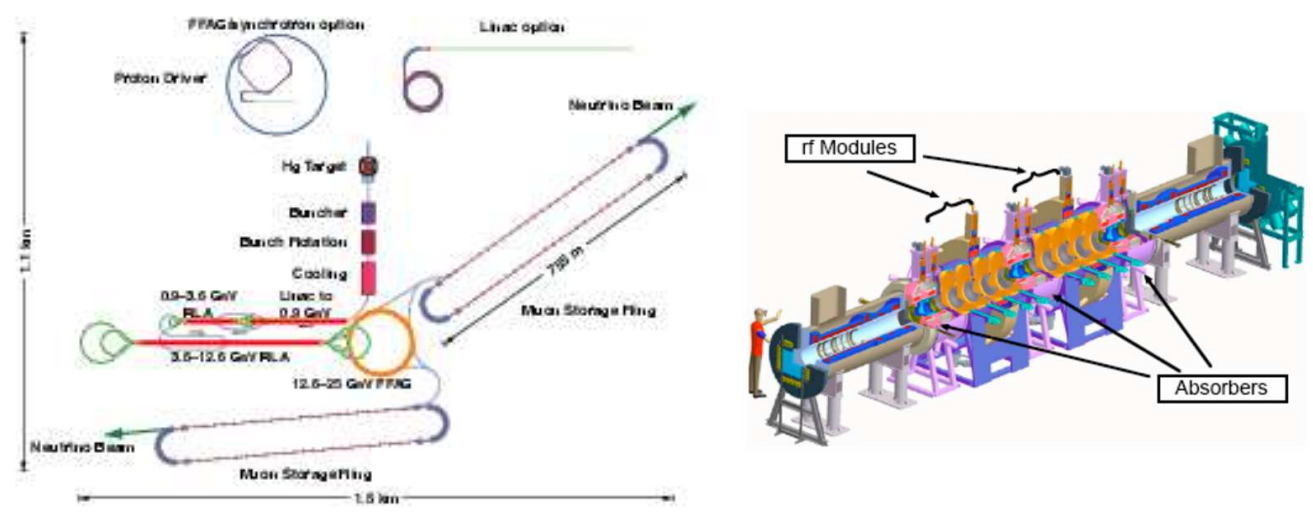

Figure 1: Left panel: schematic layout of a Neutrino Factory (IDS baseline). Right panel: layout of the MICE experiment at RAL. The secondary $\mu$ beam from ISIS enters from the lower left.

After the on-going long baseline $v$ experiments (LBL) to confirm the atmospheric $v$ claim, the next step will be to determine the small parameters $\theta_{13}, \Delta m_{21}^{2}$ and the CP violating phase $\delta$. In this context a vigorous $R \& D$ program ( $v \mathrm{~F}$, conventional superbeams and $\beta$-beams) is needed.

\section{The Neutrino Factory project.}

The neutrino factory $(v F)$ is a muon storage ring where decaying muons produce collimated neutrino beams along its straight sections. Several $v \mathrm{~F}$ designs have been proposed, such as the ones of references [1, 2]: the IDS design is shown in Figure 1 (left panel). A high intensity beam accelerated by a high power proton driver produces in a thin $\mathrm{Hg}$ target, after some accumulation and bunch compression, low energy pions. After a collection system, muons are cooled before acceleration up to 20-50 GeV/c, depending on the design. Accelerated muons of well defined charge and momentum are then injected into an accumulator where they circulate until they decay, giving two neutrino beams along the straight sections. For the design of a $v \mathrm{~F}$ some key points have to be clarified with dedicated R\&D experiments, in particular targetry, muon cooling and accelerator R\&D. The baseline option for a $v \mathrm{~F}$ target is a $\mathrm{Hg}$ jet target with impinging particles at energies $10 \pm 5$ $\mathrm{GeV}$. Available data are very scarce and for the tuning of the $\mathrm{MC}$ simulations of the $v \mathrm{~F}$ beamline the HARP data on heavy targets, such as $\mathrm{Ta}$ or $\mathrm{Pb}$, are of utmost importance [3]. In a $v \mathrm{~F}$, the produced pions are then collected through a magnetic horn or focussed through a superconducting solenoid (IDS baseline design). The MERIT (MERcury Intense Target) experiment at CERN [4] has studied the feasibility of a mercury-jet target for a $4 \mathrm{MW}$ proton beam with solenoidal pion capture, obtaining positive results.

The cooling of muons (accounting for $\sim 20 \%$ of the $v F$ final costs) increases its performances up to a factor 10. Due to their short lifetime $(2.2 \mu \mathrm{s})$, novel methods such as the ionization cooling [5] must be used. The MICE experiment [6] at RAL aims at a systematic study of one cell of the US Feasibility Study 2 cooling channel (see figure 1 for its layout). The MICE experiment will be done in stages and is expected to be finished by 2012 . 


\section{Conventional superbeams and $\beta$-beams}

Conventional neutrino superbeams [7] exploit intense proton sources to produce v's from pion decay. Open problems include targetry (mainly target heating and thermal shock) and the development of a multi-MW proton driver. The main limitations of a $v$ superbeam are connected to the intrinsic $v_{e}$ contamination $(\sim 1 \%)$ in the $v_{\mu} \mapsto v_{e}$ channel, the low energy of the produced neutrinos and the need of gigantic low-density far detectors, to access sub-dominant transitions to study $\theta_{13}$. A $v$ superbeam may be envisaged as the first step of a $v F$, as in the CERN SPL project.

Many ingredients similar to a $v \mathrm{~F}$ are incorporated also in the Beta beam concept. This new scheme to produce high intensity, low energy $v_{e}$ beams is based on the decay in flight of accelerated $\beta$-emitters [8]. As an example, in the decay of the radioactive ion: ${ }^{6} \mathrm{He}^{+++} \rightarrow{ }_{3}^{6} \mathrm{Li}^{+++} e^{-\overline{V_{e}}}$ the $H e^{6}$ ion can be accelerated to $\gamma=150$ giving a highly collimated, low energy $\overline{v_{e}}$ beam (divergence $\sim 7 \mathrm{mrad},<E_{v}>\simeq 581 \mathrm{MeV}$ ). Figure 2 shows from left to right the sensitivity to a non-zero value of $\sin ^{2} 2 \theta_{13}$, the sensitivity to the mass hierarchy (sign of $\Delta m_{32}$ ) and to the leptonic CP violation for the proposed facilities. Discovery limits are shown as fractions of all the possible values of the true values of the CP phase $\delta$ and $\sin ^{2} 2 \theta_{13}$, see [2] for more details.
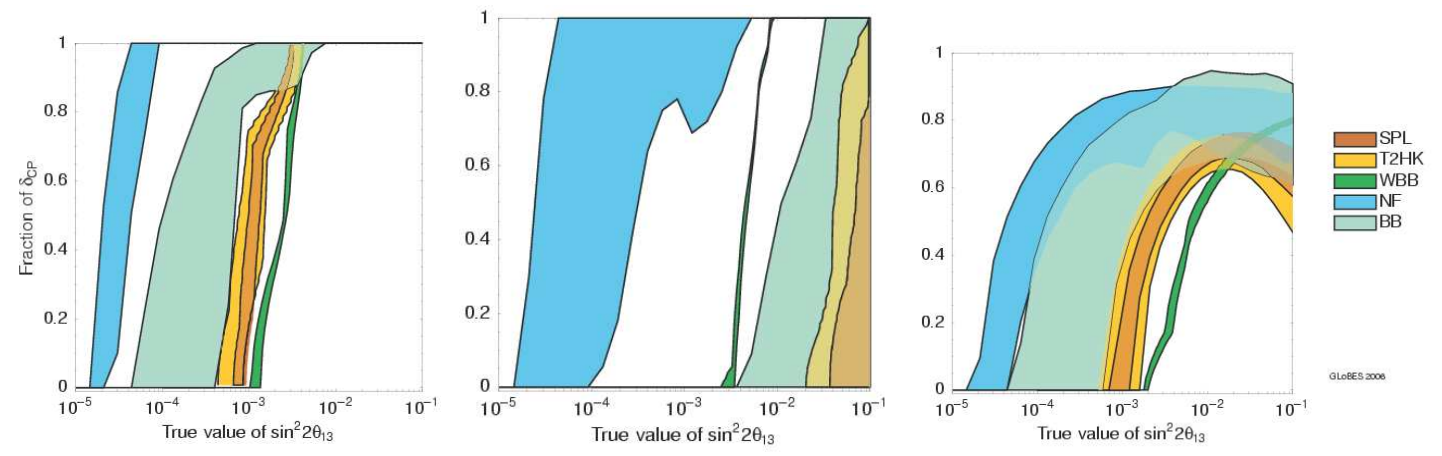

Figure 2: Comparison of physics performances for the different facilities proposed. The bands show the room for improvements as respect to the baseline option.

\section{References}

[1] M.M. Alsharo'a et al., Phys. ReV. ST. Accel. Beams 6,081001 (2003).

[2] A. Bandyopadhia et al., arXiv:0710.4947.

[3] M.G. Catanesi et al., HARP Collaboration, Phys. ReV. C77 (2008) 055207.

[4] J. Bennet et al., MERIT proposal, CERN-INTC-2004-016.

[5] A.N. Skrinsky, V.V. Parkhomchuk Sov. Jour. Nucl. Phys. 12 (1981) 3.

[6] A. Blondel et al., MICE proposal, RAL, 2004

[7] B. Richter, SLAC-PUB-8587

[8] P. Zucchelli, Phys. Lett. B532 (2002) 166 